REVISIÓN DE LITERATURA

\title{
INOCUIDAD MICROBIOLÓGICA DE LOS ALIMENTOS PARA ANIMALES EN COSTA RICA
}

\author{
Andrea Molina-Alvarado ${ }^{1}$, Fabio Granados-Chinchilla ${ }^{1}$
}

\section{INTRODUCCIÓN}

Las enfermedades transmitidas por alimentos mediadas por microorganismos patógenos o toxinas microbianas son un problema de salud pública importante. Las enfermedades de transmisión alimentaria han sido definidas por la Organización Mundial de la Salud como una enfermedad infecciosa o de naturaleza tóxica causada por, o que se sospecha es causada por, el consumo de agua o alimento (World Health Organization, 1997). Este tipo de enfermedades ejercen un efecto negativo en la salud y mortalidad humana y animal: solamente en Estados Unidos se estima que las enfermedades transmitidas por alimentos causadas por microorganismos rondan los millones, causando miles de muertes y un cargo económico cerca de 5 billones de dólares anualmente (CAST, 1994; Mead et al., 1999).Se estima que mundialmente cerca de 1,5 billones de casos de diarrea y más de 3 millones de muertes ocurren en niños menores de 5 años, y una proporción importante resultan del consumo de alimentos contaminados con patógenos y toxinas (World Health Organization,1997).

La producción y mantenimiento de la inocuidad del suministro de alimentos depende de una comprensión de las propiedades de virulencia de los organismos capaces de contaminar el alimento. Los alimentos pueden considerarse como ambientes selectivos que permiten la supervivencia de ciertos grupos de microorganismos.

Por ejemplo, las aflatoxinas que son toxinas producidas por hongos filamentosos como producto de su metabolismo secundario son también contaminantes muy importantes de los alimentos para animales. Las aflatoxinas $A F B_{1}$ y $A F G_{1}$ son producidas por hongos ubicuos (naturales del ambiente) principalmente por Aspergillus parasiticus, $A$. flavus y A. nomius (Cattaneo et al. 2011; Fallah et al, 2011). La producción de estas toxinas se ve favorecida en climas tropicales y subtropicales (Bbosa et al. 2013; Britzi et al. 2013) similares al que se tiene en Costa Rica. Estas toxinas generan varios

\footnotetext{
${ }^{1}$ Universidad de Costa Rica. San José, Costa Rica, Centro de Investigación en Nutrición Animal. San José, Costa Rica. Autor para correspondencia: andrea.molina@ucr.ac.cr 
problemas tanto a humanos como a los animales. En estos últimos por ejemplo, se han reportado problemas a nivel reproductivo, inmunológico, de ganancia de peso, entre otros. Adicional a esto se sabe que son altamente cancerígenas, teratogénicas (Bbosa et al. 2013; Sultana et al. 2009) y de difícil tratamiento para ser eliminadas de los alimentos ya que son termoresistentes (Mohammadi, 2011; Sanli et al, 2012), por lo que no se desactivan con tratamientos térmicos y permanecen en los alimentos por largos periodos de tiempo (Bosco y Mollea, 2012; Galvano et al. 1998). La presencia de aflatoxinas en los alimentos para animales no solo es de suma importancia para la salud, bienestar y productividad animal si no para la salud pública. Las aflatoxinas $A F M_{1}$ y $A F M_{2}$ son moléculas derivadas del metabolismo de la $A F B_{1}$ y $A F B_{2}$ en el hígado como parte de la detoxificación de la molécula y son eliminadas del cuerpo, en vacas, a través de la orina, las heces y la leche (Bbosa et al., 2013; Zarba et al., 1992). La $\mathrm{AFM}_{1}$ es producida en mayor proporción que la $\mathrm{AFM}_{2}$ por lo que la primera es la de mayor interés y preocupación en la leche y sus derivados.

Adicionalmente, algunos compuestos de relevancia veterinaria merecen atención desde el punto de vista económico y de salud en especial debido a que son empleados a nivel mundial. Entre estos compuestos se incluyen las penicilinas, las sulfonamidas, las tetraciclinas, las fluoroquinolonas, las aminas simpatomiméticas y las hormonas.

Los residuos de antibióticos en alimentos y en el medio ambiente son un factor determinante que aún sigue siendo omitido y usualmente no se entiende en su totalidad cuando se discute salud pública e inocuidad alimentaria. Información epidemiológica, toxicológica y química sobre antibióticos debe presentarse con el fin de evidenciar consecuencias agudas y crónicas sobre la salud de animales y humanos como resultado de los compuestos padre o de sus metabolitos.

Los compuestos veterinarios son utilizados extensivamente en animales de producción con fines terapéuticos, prevención de enfermedades infecciosas, promoción del crecimiento y la eficacia alimentaria. Todos los antibióticos aprobados para uso en países desarrollados deben apegarse a una serie de estándares de inocuidad, eficacia y calidad. Cuando se consideran productos veterinarios para uso de animales productores de alimentos, evidencia científica coherente debe presentarse con el fin de que estos cumplen el efecto deseado sin generar otros efectos perjudiciales. Estos compuestos para uso agropecuario deben regularse de forma extensiva en cada país. 
Molina y Granados. Inocuidad microbiológica de los alimentos para animales en Costa Rica

Es crucial que se enfatice en el uso prudente de antimicrobianos no solamente para minimizar su resistencia, sino para asegurar la continua eficacia, la disponibilidad de estos productos y minimizar sus residuos y su impacto sobre la salud humana.

La implementación de medidas de seguridad en granjas, un control adecuado y su registro, identificación de animales, buenas prácticas de manejo de animales, la aplicación del antibiótico y dosis correctas y la promoción de un uso juicioso y prudente de antibióticos puede cooperar mucho en aumentar la vida de uso de los antibióticos.

En esta revisión se analizan 3 contaminantes distintos que afectan directamente la inocuidad alimentaria y que están siendo investigados constantemente en Costa Rica y forman parte de programas de vigilancia a nivel nacional e internacional. Los mismos debido a su importancia en salud humana y animal han sido investigados en alimentos para animales comercializados en Costa Rica por investigadores del Centro de Investigación en Nutrición Animal (CINA) en conjunto con otros grupos de investigación del país y los resultados más relevantes de estas investigaciones serán discutidos brevemente en esta revisión.

\section{Presencia de Salmonella en alimentos balanceados y materias primas}

Salmonella es una bacteria Gram-negativa capaz de infectar humanos y una gran variedad de animales vertebrados. Las infecciones por Salmonella en humanos y animales van desde infecciones asintomáticas hasta enfermedades sistémicas y muerte (Lahiri et al., 2010; WHO Fact sheet N¹39, 2013; Hugas and Beloeil, 2014).

Salmonella es uno de los causantes principales de enfermedades de origen alimentario a nivel mundial (Majowicz et al. 2010; Scallan et al. 2011; WHO Fact sheet $\left.N^{\circ} 139,2013\right)$. Salmonellosis en humanos es generalmente causada por el consumo de alimentos contaminados de origen animal como huevos, carne y leche (WHO Fact sheet $\left.N^{\circ} 139,2013\right)$. Los casos humanos de infección por Salmonella en Europa están principalmente relacionados al consumo de huevos y carne de aves contaminados (EFSA and ECDC, 2014). Las aves y los productos para consumo humano derivados de estas son una de las principales fuentes de Salmonella en USA (Andino et al. 2014; Barbour, et al. 2015). En un estudio de Salmonella en una planta procesadora de pollo para consumo humano en Costa Rica llevado a cabo por la Escuela de Zootecnia y la Facultad de Medicina Veterinaria de la Universidad Nacional se determinó un riesgo de 
272 casos de Salmonella al año en nuestro país debido al consumo de carne de pollo contaminada (Rivera-Pérez, 2014).

Una de las posibles fuentes de contaminación de las aves con Salmonella es a través del alimento para consumo animal. Los alimentos para consumo animal son generalmente muy secos (con $a_{w}$ muy bajo) lo cual disminuye el crecimiento de microorganismos. Salmonella tiene la capacidad de sobrevivir en los alimentos aún con muy bajos niveles de agua. Además se ha observado que la capacidad de Salmonella de sobrevivir en alimentos con baja humedad varía dependiendo de la serovariedad de Salmonella que este causando la contaminación (Hiramatsu et al. 2005; Aviles et al. 2013; Chen et al. 2014).

Se ha establecido una relación entre la contaminación con Salmonella del alimento para consumo animal y la Salmonelosis tanto en animales como en humanos, lo que indica que el alimento para consumo animal constituye una posible fuente de Salmonelosis en humanos (Jones, 2011; Pennington et al. 1968; Clark et al. 1973).

Por otro lado algunas investigaciones llegan a diferentes conclusiones principalmente debido a la falta de una relación directa entre las serovariedades de Salmonella frecuentemente aisladas en alimentos para animales, las serovariedades encontradas en pollos y las causantes de brotes de Salmonelosis en humanos (Foxcroft, 1991; Jones, 2011). A pesar de que la frecuencia de aislamiento de las serovariedades de Salmonella no es la misma a lo largo de la cadena alimentaria, muchas de las cepas aisladas en alimentos para animales tanto en otros países como en Costa Rica como parte del control oficial de alimentos para animales efectuado por el Ministerio de Agricultura y Ganadería (Molina et al. 2015, en prensa), han sido asociadas con brotes de Salmonelosis en humanos a nivel nacional e internacional (Bolaños et al. 2014).

En el estudio realizado por nuestro grupo de investigación, se analizaron un total de 1725 muestras que incluye alimento balanceado y materias primas de donde se recuperaron 110 cepas identificadas como Salmonella [76 de alimento para pollo, 23 de harina de carne y hueso $(\mathrm{HCH}), 3$ alimentos para mascotas y 8 de otros alimentos] lo que corresponde a un porcentaje general de prevalencia de $6,4 \%$, entre los años 2009 y 2014. En términos generales, los aislamientos de Salmonella fueron recuperados en su mayoría de alimentos para pollo (en distintas fases) y harina de carne y hueso. Estas últimas mostraron una mayor prevalencia relativa (Molina et al. 2015, en prensa). De estas cepas aisladas, las serovariedades más frecuentemente encontradas se describen en la Figura 1. 
Molina y Granados. Inocuidad microbiológica de los alimentos para animales en Costa Rica

Las harinas de origen animal como harina de carne y hueso $(\mathrm{HCH})$, tortave y harina de pescado, las cuales son elaboradas a partir del producto de descarte de la industria de carne de res, pollo y pescado respectivamente, han mostrado tener internacionalmente una alta prevalencia de Salmonella (Veldman et al, 1995; Jones y Richardson, 2004; Norwegian Scientific Commite for Food Safety, 2006).

La prevalencia en $\mathrm{HCH}$ es relevante debido a que ha encontrado uso como una fuente relativamente económica y disponible de proteína (Hendriks et al. 2002) y se incorpora en las formulaciones de alimento con el fin de mejorar el perfil de aminoácidos del producto terminado. A pesar de que el uso de $\mathrm{HCH}$ es prohibido en la fabricación de alimentos destinados para ganado (debido al riesgo de trasmisión encefalopatía espongiforme bovina), en Costa Rica es, sin embargo, aún utilizada para alimentar monogástricos o como una fuente de bajo costo de proteína en alimentos de mascotas (Adedokun and Adeola, 2005; Granados-Chinchilla and Rodríguez, 2014). Actualmente existen registradas en Costa Rica 5 empresas procesadoras de harina de carne y hueso, 4 de tortave y 2 de harina de pescado.

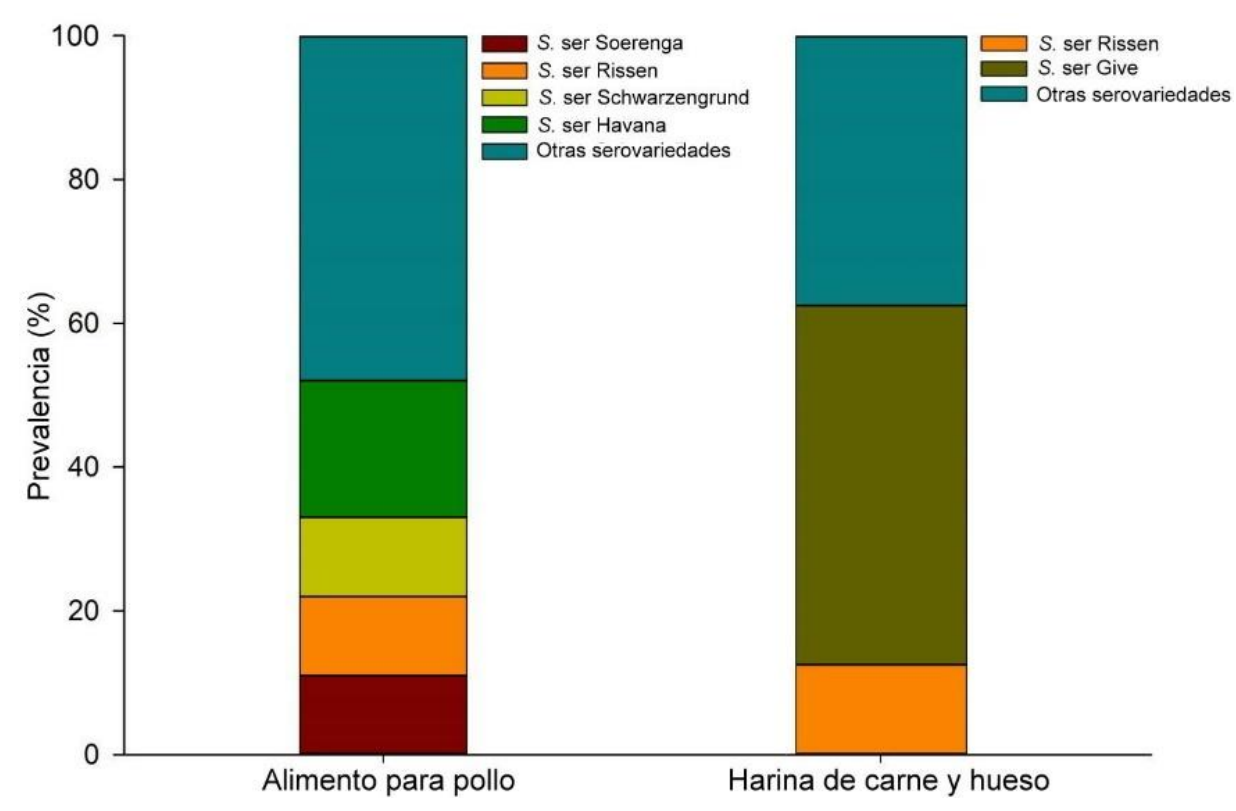

Figura 1. Serovariedades más comunes encontradas en alimentos balanceados para pollos y harina de carne y hueso.

En la elaboración de estas harinas de origen animal uno de los puntos críticos de control más importantes es la cocción a altas temperaturas, lo cual debería eliminar 
toda contaminación con Salmonella y también bacterias esporuladas como Clostridium perfringes. Los resultados de la investigación realizada en el CINA donde El 26,7 \% de las 86 muestras de $\mathrm{HCH}$ analizadas resultaron contaminadas con Salmonella, indica que las harinas se están recontaminando luego del proceso de cocción o los procesos de cocción realizados en el país no son los adecuados (Molina et al. 2015, en prensa).

\section{Presencia de Aflatoxina $M_{1}$ en leche y queso en Costa Rica}

La contaminación de leche y sus productos con aflatoxina es de extrema importancia y es un problema serio, por cuanto la especie humana y los otros mamíferos, particularmente los recién nacidos son dependientes de la leche materna como parte de una nutrición basal completa. Los infantes son por lo general más sensibles a las toxinas que los adultos debido a su relativo bajo peso corporal y actividad celular alta a pesar de poseer un sistema inmune parcialmente desarrollado. Una consecuencia de la biotransformación lenta en el organismo de los infantes, podría ser un tiempo de circulación mayor del contaminante. La IARC (1993) categoriza la AFM $_{1}$ como un posible cancerígeno. La Unión Europea y el Codex Alimentarius han fijado el límite de $\mathrm{AFM}_{1}$ de ingesta a un máximo de $50 \mathrm{ng} / \mathrm{kg}$ en productos lácteos mientras que la FDA ha establecido un límite de 500 ng/kg. En Costa Rica los límites se establecieron de acuerdo al Codex antes de mediados del 2014 en 50 ng $A F M_{1} / L$ leche $\left(N^{\circ} 33812\right.$ MEIC-MAG-S; RTCR: 401-2006) después del 2014 se estableció como límite 500 ng/L (337-2014, RTCA 67.04.66:12).

Comparada a la $\mathrm{AFB}_{1}$, la $\mathrm{AFM}_{1}$ es menos cancerígena y mutagénica, sin embargo, se ha reportado que exhibe un gran nivel de genotoxicidad en los animales (JECFA 2001). Varias investigaciones, realizadas en países con semejanzas culturales, un nivel sociocultural similar y que comparten características geográficas como clima, manejo de cultivos como los que poseen los países latinoamericanos, han reportado la presencia de $\mathrm{AFM}_{1}$ en leche con resultados similares a los obtenidos en Costa Rica. Un estudio brasileño (Garrido et al. 2003) determinó niveles de 50-500 ng/L en 20,9\% de las muestras que analizaron. Un grupo de investigación colombiano (Díaz y Espitia 2006) encontraron una prevalencia de un $70 \%$ de muestras con concentraciones de 10,6-290 ng/L. Un estudio Iraní reportó resultados similares un $71 \%$ de muestras positivas entre 5,8 y $528 \mathrm{ng} / \mathrm{L}$ (Fallah, 2010). Niveles menores de $\mathrm{AFM}_{1}$, en comparación, (hasta $17 \mathrm{ng} / \mathrm{L}$ ) se encontraron en un estudio argentino (López et al., 2003) y un estudio Italiano reciente (Santini et al., 2013), quienes reportaron 
Molina y Granados. Inocuidad microbiológica de los alimentos para animales en Costa Rica

concentraciones por encima de $28,5 \mathrm{ng} / \mathrm{L}$ y $18 \mathrm{ng} / \mathrm{L}$, respectivamente. Por otra parte, dos estudios independientes en México e Irán, mostraron un 50\% de incidencia de $\mathrm{AFM}_{1}$ en leche con concentraciones máximas de hasta $8.350 \mathrm{ng} / \mathrm{L}$ y $410 \mathrm{ng} / \mathrm{L}$, respectivamente (Carvajal et al. 2003; Rohani et al. 2011). La variabilidad de los resultados puede ser atribuida a los métodos analíticos empleados, sistemas de alimentación y condiciones ambientales (Galvano et al. 1996).

La contaminación de la leche con AFM 1 puede ocurrir de dos formas, a saber, de forma directa por el consumo de alimentos animales contaminados con $\mathrm{AFB}_{1} \mathrm{O}$ de forma indirecta por la contaminación de la leche y sus productos con hongos (Applebaum et al. 1982; Blanco et al. 1993; Barrios et al. 1997; Sarimehmetoolu et al. 2003; Driehuis et al. 2008; Sugiyama et al. 2008). Sin embargo, se debe hacer notar que la $A F M_{1}$ es un metabolito de la $A F B_{1}$. La contaminación de leche y derivados por la $\mathrm{AFM}_{1}$ varía de acuerdo con la ubicación geográfica y las condiciones climáticas. El acarreamiento de la toxina del alimento a la leche se estima que ronda un $(2,66 \pm$ $1,24 \%$ ) y es proporcional al rendimiento de producción, la raza, rasgos individuales y etapa de lactancia del animal (Veldman et al. 1992). La toxina se ha descrito ocurre en leche 4-5 h luego de haber alimentado a vacas lactantes con alimentos contaminado. A pesar de que la $\mathrm{AFM}_{1}$ es el compuesto hidroxilado mayoritario producto del metabolismo pueden presentarse otros compuestos similares pero en bajas concentraciones relativas (dos o tres órdenes de magnitud) como la AFM2, AFM4, AFQ o aflatóxico.

Los alimentos contaminados son los responsables principales de la contaminación en leche y productos lácteos. Por tanto, medidas de control estrictas para fabricantes locales de alimentos, así como importadores de materias primas y alimentos es un requisito para obtener niveles bajos de toxina en leche y derivados.

El metabolismo de $\mathrm{AFB}_{1}$ resulta de la función oxidasa microsomal hepática pero, dependiendo de la especie, otras conversiones metabólicas son posibles. Por otra parte, operaciones unitarias o procesos tecnológicos incluyendo la pasteurización o esterilización no poseen efecto sobre la concentración o actividad de la molécula. En la leche no existe una distribución homogénea de la toxina. Debido a la naturaleza semipolar de la $\mathrm{AFM}_{1}$ de la molécula la mayoría se une a la caseína y $30 \%$ se estima se asocia con sólidos no grasos.

Sin embargo, para el caso de derivados lácteos como el queso, la evidencia sugiere que la fracción que se retiene por la caseína u otras proteínas lácteas es dependiente del proceso de manufactura. De hecho, de forma indirecta se ha demostrado que el 
queso fresco fabricado en Costa Rica, muestra concentraciones significativamente menores a las encontradas en leches comerciales. Específicamente, luego de analizar muestras de leche fluida ( $2 \mathrm{~g} / 100 \mathrm{~mL}$ grasa; $n=70)$, queso fresco $(n=70)$ y natilla $(n$ $=70)$ recolectadas de distintos supermercados se encontraron concentraciones de $\mathrm{AFM}_{1}$ entre los 19 hasta los $629 \mathrm{ng} / \mathrm{Ly}$ desde 31 hasta $276 \mathrm{ng} / \mathrm{L}$, con valores promedios de $136 \mathrm{ng} / \mathrm{L}$ y $74 \mathrm{ng} / \mathrm{L}$, de forma respectiva. Por otro lado, ninguna de las 70 muestras de natilla que se analizaron fueron positivas para $\mathrm{AFM}_{1}$ (Chavarría et al. 2015). El detalle de estos resultados se muestra en la Figura 2.

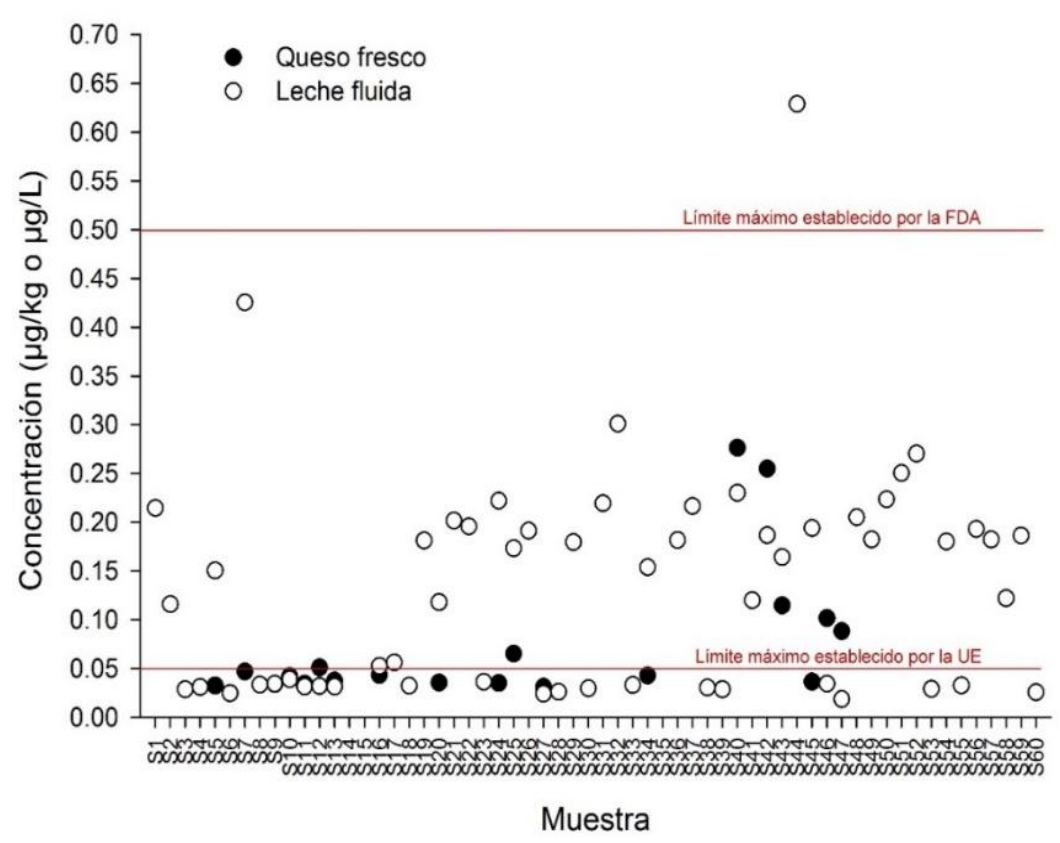

Figura 2. Distribución de las concentraciones de $A F M_{1}$ en muestras comerciales de leche y queso fresco.

Por otra parte, la interacción de la $\mathrm{AFM}_{1}$ con proteínas incluso ha demostrado tener también consecuencias analíticas. Considerando que las recuperaciones en matrices lácteas producto de la fermentación como queso y natilla eran menores a las esperadas utilizando extracción directa con metanol y simultáneamente como un esfuerzo para reducir el uso de disolventes orgánicos (especialmente clorados), utilizados usualmente durante el proceso de extracción de $A F M_{1}$, nuestro grupo de investigación hipotetizó que las proteínas lácteas y las grasas jugaban un papel 
Molina y Granados. Inocuidad microbiológica de los alimentos para animales en Costa Rica

preponderante en el secuestro de $\mathrm{AFM}_{1}$ y por ende su contacto con el disolvente de extracción. Esto se comprobó al aumentar las recuperaciones analíticas de forma dramática cuando se utilizaron enzimas proteolíticas y lipolíticas como parte del pretratamiento de la muestra (Chavarría et al. 2015). Más tarde de forma independiente otro grupo de investigadores (Pierti et al. 2015) utilizaron digestión por pepsina de manera similar y corroboraron aún más nuestros hallazgos. Un resumen gráfico de la metodología utilizada por Chavarría y colaboradores se muestra en la Figura 3.

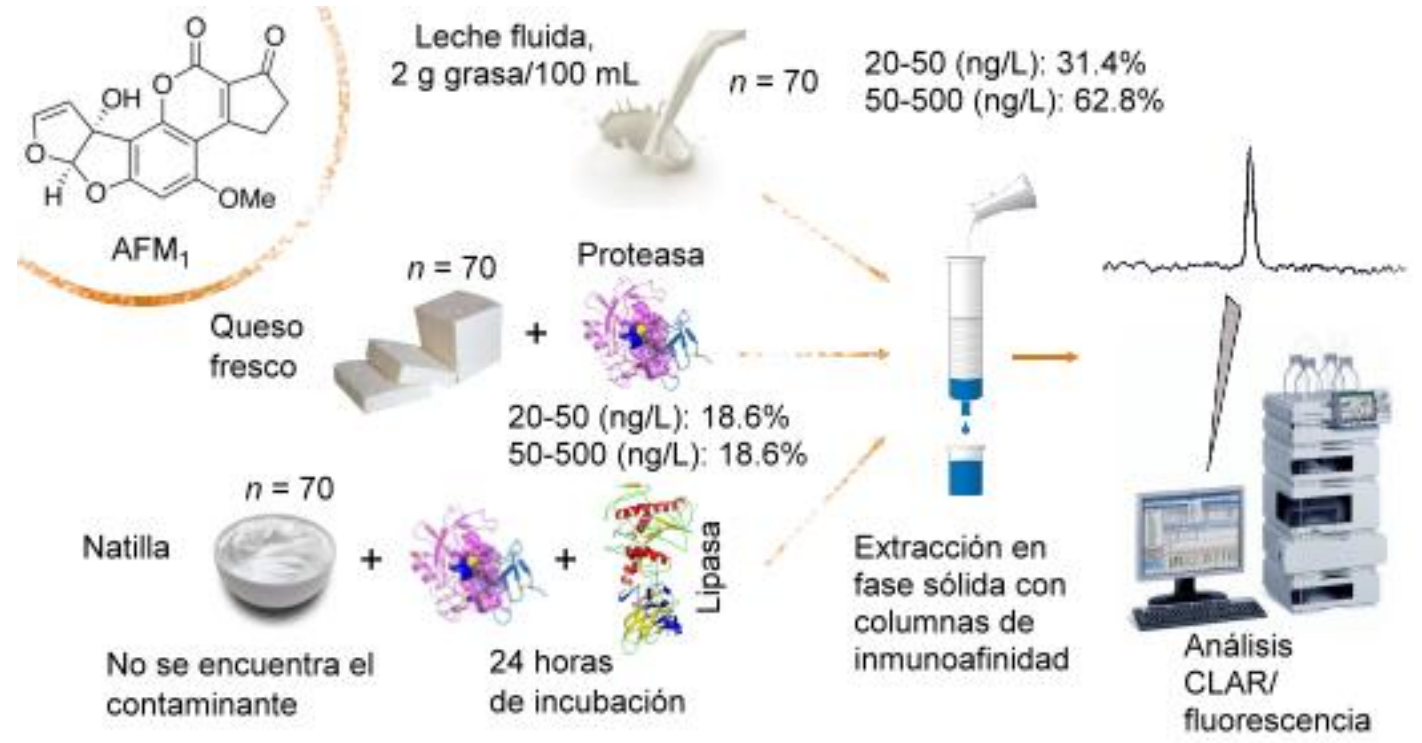

Figura 3. Representación/resumen gráfico de la metodología diseñada en el CINA para la cuantificación de $\mathrm{AFM}_{1}$ en muestras comerciales de leche, queso fresco y natilla. Se destacan además los hallazgos más importantes

Comparada a la leche cruda, la prevalencia de $\mathrm{AFM}_{1}$ en leche comercial es usualmente mayor, resultando de la adición de solo algunas muestras contaminadas al bulto que no posee contaminación. Sin embargo, por la misma razón las leches comerciales en general muestran niveles más bajos de $\mathrm{AFM}_{1}$.

Finalmente, la transmisión en leche de otras toxinas que se pueden encontrar en alimentos balanceados, como deoxinivalenol, fumonisinas, ocratoxina $A$, sterigmatocyna, toxina T-2 y zearalenona, es poco probable. 


\section{Determinación de tetraciclinas y bacterias resistentes en alimentos para animales}

En los alimentos balanceados se han encontrado además de compuestos padre de antibióticos presencia de metabolitos y otros compuestos propios de su degradación. Un ensayo realizado en laboratorio demuestra que durante el almacenamiento de un alimento medicado de concentración conocida, a una temperatura cercana a la ambiente y con una humedad relativa elevada (condiciones que reúnen muchas regiones de nuestro país) la concentración inicial de clortetraciclina decae significativamente (Figura 4). Esto se ve empeorado por el hecho que las tetraciclinas suelen ser sensibles a la luz.

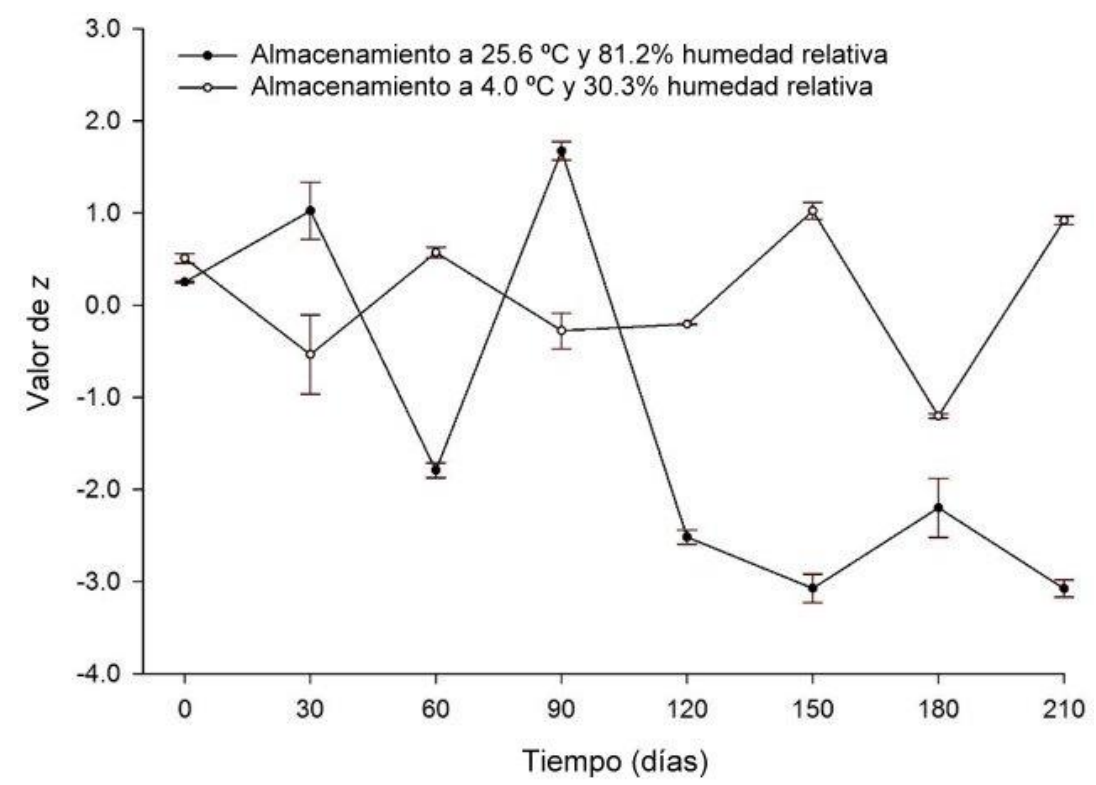

Figura 4. Comportamiento de la clortetraciclina durante dos condiciones distintas de almacenaje de una alimento medicado.

En el CINA se desarrolló un método para determinación simultánea de tetraciclinas y macrólidos en materias primas y alimentos para animales (Granados-Chinchilla et al. 2012) ya que estos antibióticos se presentan frecuentemente en dichas matrices y no existen muchos métodos que respondan a esta necesidad. Nuestro método acopla una extracción orgánica y una extracción de limpieza en fase sólida con una 
Molina y Granados. Inocuidad microbiológica de los alimentos para animales en Costa Rica

separación de cromatografía liquida de alta resolución (HPLC) y una derivatización post columna no acuosa todo bajo los principios de química verde. Este nuevo método detecta desde $0,2 \mu \mathrm{g} \mathrm{kg}-1$ of tetraciclina, oxitetraciclina, chlortetraciclina, doxiciclina, tigeciclina, and 4-epitetraciclina en esta matriz compleja y heterogénea. Minociclina y tilosina pueden también ser detectadas por nuestro método pero usando espectrofotometría ultravioleta y con limites de detección mínimo y máximo de 1,5 y $1,9 \mathrm{mg} / \mathrm{kg}$ respectivamente. Los coeficientes de correlación lineal se encontraron entre 0,996 y 0,999 para todos los antibióticos analizados en el rango de concentración entre $0,5-10 \mathrm{mg} / \mathrm{kg}$. Los porcentajes de recuperación para tetraciclina y tilosina se encuentran entre 59 y $97 \%$ y entre 98 y $102 \%$ respectivamente. La desviación estándar de replicas esta debajo del $5 \%$.

Debido a esto el CINA cuenta actualmente con un nuevo método con el cual es posible detectar simultáneamente antibióticos del grupo de los macrólidos y de las tetraciclinas con una alta especificidad y sensibilidad (Granados-Chinchilla et al. 2012). Con este método se analizaron 20 alimentos para animales vendidos en Costa Rica, se detectaron inconsistencias con respecto a la etiqueta, mezclas prohibidas de tetraciclinas así como concentraciones de tetraciclinas que infringen regulaciones internacionales (Granados-Chinchilla et al. 2012).

Los antibióticos alteran la homeostasis de las comunidades microbianas y seleccionan bacterias resistentes a los antibióticos en la naturaleza. La acumulación de altas concentraciones de antibióticos en el medio ambiente debido a actividades humanas como la agricultura, la producción pecuaria, entre otros representan una contaminación muy importante especialmente en países con una economía basada en la agricultura y la producción pecuaria como Costa Rica. Para la toma de decisiones con respecto a planes de contingencia, políticas o reglamentaciones tendientes a disminuir el problema de resistencia a antibióticos es necesario tener información cualitativa y cuantitativa sobre el uso de antibióticos en las diferentes actividades humanas en el país y principalmente en zonas con alta actividad agrícola y pecuaria. Actualmente la información de este tipo en el país sigue siendo escasa.

En un estudio realizado por un grupo de investigadores del CINA en conjunto con investigadores del Centro de Investigaciones en Enfermedades Tropicales (CIET) y del Instituto Regional de Estudios en Sustancias Tóxicas (IRET) de la Universidad Nacional de Costa Rica en la zona de riego Arenal Tempisque, la cual es una zona con una gran actividad agrícola y pecuaria, se caracterizó el peligro asociado a los antibióticos usados en agricultura, acuacultura, porcicultura y medicina veterinaria y 
humana durante el 2008. Este estudio revela que en esa zona se usaron 38 antibióticos distintos pertenecientes a 15 familias diferentes y el consumo de antibióticos fue estimado entre 1.169-109.908 $\mathrm{g}$ ha año, casi la mitad de esto correspondió a fenicoles. La oxitetraciclina fue el antibiótico más utilizado en agricultura y en medicina veterinaria. Oxitetraciclina, florfenicol, clortetraciclina, sulfametoxazol, eritromicina, ciprofloxacina, enrofloxacina, sulfametazina, trimetoprim y tilosina, en ese orden mostraron los indicadores de peligrosidad más altos en este estudio. Laoxacilina, doxiciclina, oxitetraciclina, sulfametazina, and ciprofloxacina presentaron coeficientes de peligrosidad relativamente altos (De la Cruz et al. 2014).

En la acuacultura la tetraciclina es usada comúnmente ya sea agregada en el alimento o directamente en los estanques con el fin de prevenir o tratar infecciones bacterianas.

Debido a la degradación de los antibióticos por mecanismos bióticos y abióticos es común encontrar concentraciones de metabolitos; en el caso de las tetraciclinas, epi, iso y anhidro tetraciclinas. Estos metabolitos de las tetraciclinas son biológicamente activos. Aunado a esto, los estándares de grado alimentario de tetraciclinas que se utilizan para dosificar esto por cuanto son productos del metabolismo de actinobacterias (por ejemplo género Streptomyces) por lo que además del compuesto padre deseado se obtienen subproductos y las subsecuentes etapas de purificación de estos materiales no son completamente efectivas. Por ejemplo, Un alimento medicado con un estándar 95\% de pureza a una concentración de $400 \mathrm{mg} / \mathrm{kg}$ produce una contaminación de $20 \mathrm{mg} / \mathrm{kg}$ del subproducto. Este problema se agrava si se considera el acarreamiento de los antibióticos durante su fabricación entre lotes medicados y aquellos que no le están. De hecho, un grupo de investigación en Holanda demostró que de 140 muestras de lavado entre lotes obtenidas de 21 fábricas de alimentos el $87 \%$ de estas muestras poseían residuos de antibióticos con concentraciones que variaban entre los $0,1-154 \mathrm{mg} / \mathrm{kg}$ y que el acarreamiento es especialmente importante para la oxitetraciclina (Stoker et al. 2013).

Finalmente, los compuestos padre y los metabolitos o subproductos que se obtienen durante la degradación de estos antibióticos tienen una efecto sobre poblaciones de bacterias que no necesariamente corresponden a los microorganismos diana.

Con el fin de determinar los efectos de la tetraciclina y uno de sus epímeros reversibles, la 4-epitetraciclina, sobre la función y estructura de la comunidad microbiana presente en un sedimento proveniente de una granja de tilapia de nuestro país, se evaluaron los perfiles fisiológicos a nivel de comunidad y los perfiles de ácidos 
Molina y Granados. Inocuidad microbiológica de los alimentos para animales en Costa Rica

grasos y fosfolípidos de membrana que se obtuvieron del microcosmos expuesto durante un lapso de 12 días a 5, 10, 50, o $75 \mathrm{mg} \mathrm{kg}^{-1}$ de estos antibióticos.

La flora predominante en este sedimento fueron bacterias Gram-negativas, las variaciones encontradas en ambos perfiles analizados luego de la exposición a estos antibióticos indican que concentraciones de tetraciclina $>50 \mathrm{mg} / \mathrm{kg}$ de tetraciclina favorecen el crecimiento de hongos y levaduras en dicho sedimento y ambos antibióticos a concentraciones $<10 \mathrm{mg} / \mathrm{kg}$ seleccionan microorganismos con un marcador biológico (11:0 iso $3 \mathrm{OH})$ ligado a bacterias intrínsecamente resistentes a antibióticos y desinfectantes pertenecientes al género Xanthomonas (GranadosChinchilla et al. 2013). Los autores concluyen que concentraciones elevadas de tetraciclina y 4-epitetraciclina (uno de los productos de la degradación de la tetraciclina) que son relevantes a nivel de campo tienen el potencial de modificar la composición y la función de una comunidad microbiana de sedimento. Este estudio resalta la importancia de considerar productos de degradación en investigaciones ecotoxicológicas (Granados-Chinchilla et al. 2013).

Como ya hemos expuesto diversas tetraciclinas son usadas en las diferentes producciones pecuarias con el fin de prevenir o tratar infecciones bacterianas. Debido a esto en el 2014 un grupo de investigadores del CINA y del Centro de Ciencia y Tecnología (CITA) se dieron a la tarea de analizar si la dosis de antibióticos adicionada en los alimentos para animales definía la cantidad de bacterias resistentes a los antibióticos en estos alimentos, para esto se determinaron las concentraciones de oxytetraciclina (OTC), doxyciclina, tetraciclina and chlortetraciclina sus epímeros así como la cantidad de bacterias resistentes a la oxitetraciclina y sus nivel de resistencia en alimentos para peces, aves, cerdos y camarones (21 muestras de cada tipo de alimento fueron analizadas) comercializados en Costa Rica (Granados-Chinchilla et al. 2014).

Las mayores concentraciones de tetraciclina (119-8.365 mg/kg), así como la proporción más grande de bacterias resistentes a $10 \mu \mathrm{g} / \mathrm{ml}(1,8-92,4 \%)$ o $100 \mu \mathrm{g} / \mathrm{ml}$ of OTC $(12,5-63,8 \%)$ se presentaron en el alimento para peces. En el alimento para aves y cerdos se encontraron concentraciones intermedias de tetraciclina $(78-438 \mathrm{mg} / \mathrm{kg}$ ) y (41-1.076 $\mathrm{mg} / \mathrm{kg})$ y bacterias resistentes de 0,2-66\% y 0,3-49\%, respectivamente. El alimento para camarones presento los niveles más bajo de tetraciclinas (21,5- 50,3 $\mathrm{mg} \mathrm{kg}^{-1}$ ), ausencia de oxitetraciclina así como ausencia de bacterias resistentes a este antibiótico. Sin embargo, algunos residuos fueron identificados como doxiciclina, un 
antibiótico que aún está reservado para terapia humana y solamente aplicaciones específicas en animales domésticos.

Los valores de concentración inhibitoria mínima para oxitetraciclina para 150 aislamientos a parir de alimentos para pescado y pollo fueron $>256 \mu \mathrm{g} / \mathrm{ml}$, mientras que los aislamientos para cerdos exhibieron una concentración de $192 \mu \mathrm{g} / \mathrm{ml}$. Esto evidencia una población bacteriana con una muy alta resistencia a las tetraciclinas.

La mayoría de las bacterias resistentes a la oxitetraciclina eran bacterias Grampositivas pertenecientes a los géneros Staphylococcus y Bacillus. En esta investigación se logro demostrar en alimento para peces que existe una correlación entre la dosis de oxitetraciclina en el alimento y la colonización de bacterias resistentes a este antibiótico en el alimento. A pesar de esto se encontraron grandes poblaciones de bacterias resistentes en alimentos para peces, cerdos y aves que no estaban medicados lo que sugiere que las materias primas y el proceso de manufactura puede influenciar el transporte de bacterias resistentes a la oxitetraciclina en alimentos para animales (Granados-Chinchilla et al. 2014). Estos resultados son preocupantes ya que demuestran que todos los factores necesarios para la emergencia y diseminación a través de la cadena alimenticia de la resistencia a antibióticos están presentes simultáneamente en las producciones pecuarias nacionales (Granados-Chinchilla et al. 2014).

Un aspecto muy importante respecto a la adición de tetraciclinas en los alimentos para animales es la posible interacción entre este antibiótico y los componentes propios de dichos alimentos como proteínas y calcio, alterando sus propiedades y función. Este es un aspecto que en la mayoría de los casos no es tomado en consideración a la hora de dosificar estos alimentos. Debido a esto dos investigadores del CINA y del CIET se dieron a la tarea de analizar el impacto de las concentraciones de proteína cruda y calcio sobre la biodisponibilidad de 14 tetraciclinas de primera, segunda y tercera generación, epímeros de tetraciclina y otros derivados en 84 alimentos de camarones, cerdos, tilapia y aves comercializados en Costa Rica (Granados-Chinchilla y Rodríguez, 2014).

La concentración de tetraciclinas y sus epímeros fue determinada por HPLC utilizando el método desarrollado en el CINA (Granados-Chinchilla et al. 2012), la cantidad de tetraciclinas biodisponible fue determinada con 2 biosensores distintos los cuales discriminan tetraciclina inactiva o unida de la tetraciclina libre y activa. La cantidad de 
Molina y Granados. Inocuidad microbiológica de los alimentos para animales en Costa Rica

tetraciclina biodisponible (libre y activa) fue inversamente proporcional a la concentración de proteína cruda y de calcio en los alimentos analizados.

El alimento para camarones, seguido por el de tilapia presentaron las concentraciones más altas de proteína cruda $(14,5-52,2 \mathrm{~g} / 100 \mathrm{~g})$ y de calcio $(0,89-5,90 \mathrm{mg} / \mathrm{kg})$ y como se predijo la fracción más baja de tetraciclinas biodisponibles (12-40\%). Este trabajo aporta ecuaciones que predicen la biodisponibilidad de las tetraciclinas a partir de las concentraciones de calcio, proteína cruda y tetraciclina en el alimento para animales. Esta información debería ser usada a la hora de formular alimentos medicados con el fin de usar los antibióticos de una forma más racional y eficiente y evitar tener altas concentraciones de tetraciclinas en los alimentos que al final no van a estar biodisponibles y no van a tener la función antimicrobiana esperada (GranadosChinchilla y Rodríguez, 2014).

\section{CONSIDERACIONES FINALES Y APLICACIONES}

De todas las investigaciones realizadas en el CINA discutidas en esta revisión tanto para Salmonella como para $\mathrm{AFM}_{1}$ en leche y tetraciclinas en alimentos para animales se puede concluir que el país cuenta con las metodologías para realizar un control efectivo de dichos contaminantes así como realizar investigaciones en estos temas. Sin embargo, principalmente en el caso de la resistencia a antibióticos es necesario hacer más conciencia a nivel país de la importancia de aplacar este problema tan grave y de aumentar y mejorar los programas de vigilancia y de educación sobre estos contaminantes. A pesar de que nuestra investigación se basa en grupo específico de tetraciclinas otros antibióticos probablemente presenten problemáticas similares. Estas investigaciones y otras que se están ejecutando en este momento promueven la creación de conocimiento científico local que permita buscar soluciones con el fin de disminuir la prevalencia en nuestro país de estos contaminantes y residuos. 


\section{LITERATURA CONSULTADA}

Andino, A., Pendleton, S., Zhang, N., Chen, W., Critzer, F., Hanning, I. 2014. Survival of Salmonella enterica in poultry feed is strain dependent. Poultry Science 93:441-447.

Aviles, B., Klotz, C., Smith, T., Williams, R., Ponder, M. 2013. Survival of Salmonella enterica Serotype Tennessee during simulated gastric passage is improved by low water activity and high fat content. J. Food Prot. 76:333-337.

Barbour, E.K., Ayyash, D.B., Alturkistni, W., Alyahiby, A., Yaghmoor, S., Iyer, A., Yousef, J., Kumosani, T., Harakeh, S. 2015. Impact of sporadic reporting of poultry Salmonella serovars from selected developing countries. J. Infect. Dev. Ctries. 9:001-007.

Bbosa, G.S., Kitya, D., Odda, J., Ogkwal-Okeng, J. 2013. Aflatoxins metabolism, effects on epigeneticmechanisms and their role in carcinogenesis. Health. 5:14-34.

Bolaños, H.M., Acuña, M.T., Tijerino, A., Jiménez, A., Duarte, F., Sánchez, L.M., Dittel, I.,Vargas, J.L., Campos, E. 2013. Red Nacional de Laboratorios de Bacteriología. 2014. Informe de vigilancia basada en laboratorio: Vigilancia basada en laboratorio de Salmonella, Costa Rica, 2013. INCIENSA. Disponible en: http://www.inciensa.sa.cr

Britzi, M., Friedman, S., Miron, J., Solomon,R., Cuneah, O., Shimshoni,J.A., Soback,S., Ashkenazi,R., Armer, S., Shlosberg, A.2013. Carry-over ofaflatoxin B1 to aflatoxin M1 in high yielding Israeli cows in mid- and latelactation. Toxins. 5:173-183.

CAST (Council of Agricultural Science andTechnology). 1994. Foodborne Pathogens: Risksand Consequences. Task Force Report No. 122, Council of Agricultural Science and Technology,Ames, lowa.

Cattaneo, T.M., Marinoni, L., Barzaghi, S., Cremonesi, K., Monti, L. 2011. Testing the suitability ofdifferent high-performance liquid chromatographicmethods to determine aflatoxin M1 in a soft freshltalian cheese. J Chrom A. 1218:4738-4745.

Chen, W., Golden, D.A., Critzer, F.J. 2014. Salmonella survival and differential expression of fatty acidbiosynthesis-associated genes in a low-water-activity food. Lett Appl Microbiol. 59:133-138.

Clark, G.M., Kaufmann, A.F., Gangarosa, E.J., Thompson, M.A. 1973. Epidemiology of an international outbreak of Salmonella agona. Lancet 2:490-493. 
de la Cruz, E., Fournier, M.L., García, F., Molina, A., Chavarría, G., Alfaro, M., Ramírez, F., Rodríguez, C. 2014. Hazard prioritization and risk characterization of antibiotics in an irrigated tropical regionused for intensive crop, livestock, and aquaculture farming. J Environ Biol. 35:85-98.

European Food Safety Authority (EFSA), European Centre for Disease Prevention and Control (ECDC). 2014. The European Union Summary Report on Trends and Sources of Zoonoses, Zoonotic Agents and Food-borne Outbreaks in 2013. EFSA Journal. 12:3547.

Fallah, A.A. 2010. Aflatoxin $\mathrm{M}_{1}$ contamination in dairy products marketed in Iran during winter and summer. Food Control, 21:1478-1481.

Foxcroft, P.D. 1991. A European view of animal by-products in feed. Pages 93-96 in Proc. 26th Natl. Mtg. Poult. Health Condemnations. Delmarva Poult. Ind. Inc., Ocean City, MD.

Granados-Chinchilla F, Alfaro M, Chavarría G, Rodríguez C. 2014. Unravelling a vicious circle: animal feed marketed in Costa Rica contains irregular concentrations of tetracyclines and abundant oxytetracycline-resistant Gram-positive bacteria.Food Addit Contam Part A Chem Anal Control Expo Risk Assess. 31:1017-25.

Granados-Chinchilla, F, Arias-Andrés, M, Rodríguez, C. 2013. Tetracycline and 4epitetracycline modified the in vitro catabolic activity and structure of a sediment microbial community from a tropical tilapia farm idiosyncratically. J Environ Sci Health B. 48:291-301.

Granados-Chinchilla, F., Sánchez, J., García, F., Rodriguez, C. 2012. A novel green chemistry method for non-aqueous extraction and HPLC detection of first-, second-, and thirdgeneration tetracyclines, 4-epitetracycline, and tylosin in animal feeds. J. Agric. Food Chem. 60:7121-7128.

Granados-Chinchilla, F.; Rodriguez, C. 2014. Bioavailability of in-feed tetracyclines is influenced to a greater extent by crude protein rather than calcium. Anim. Feed Sci. Technol. 198, 323-332. 
Hiramatsu, R., Matsumoto, M., Sakae, K. and Miyazaki, Y. 2005. Ability of Shiga toxinproducing Escherichia coli and Salmonella spp. to survive in a desiccation model system and in dry foods. Appl Environ Microbiol. 71, 6657-6663.

Hugas M., Beloeil PA. 2014. Controlling Salmonella along the food chain in the European Union - progress over the last ten years. Euro Surveill. 19: 20804.

INCIENSA. 2013. Informe de vigilancia basada en laboratorio Agentes causantes de diarrea y otras infecciones, por región y laboratorio, 1 - 31 de mayo de 2013. Disponible en:http://www.inciensa.sa.cr/vigilancia epidemiologica/informes vigilancia/2013/Bacter ias/Agentes\%20causantes\%20de\%20diarrea\%20y\%20otras\%20infecciones, \%20mayo \%202013.pdf. Consultado en abril 24, 2015.

Jones, FT, Richardson KE. 2004. Salmonella in commercially manufactured feeds. Poult. Sci. 83:384-391.

Jones, FT. 2011. A review of practical Salmonella control measures in animal feed. J Appl Poult Res. 20:102-113.

Lahiri, A., Lahiri, A., lyer, N., Das, P., Chakravortty, D. 2010.Visiting the cell biology of Salmonella infection. Microbes Infect. 12:809-818.

Majowicz, S.E., Musto, J., Scallan, E., Angulo, F.J., Kirk, M., O'Brien, S.J., Jones, T.F., Fazil, A., Hoekstra, R.M. 2010.The global burden of nontyphoidal Salmonella gastroenteritis. Clin Infect Dis. 50:882-889.

Mead, P.S., Slutsker, L., Dietz, V., McCaig, L.F., Bresee, J.S., Shapiro, C., Griffin, P.M., Tauxe, R.V. 1999. Food-related illness and death in the United States. Emerging Infectious Diseases. 5:607-625.

Mohammadi, H. 2011. A review of Aflatoxin M1, Milk, and Milk products. In: R. G. GuevaraGonzalez ed. Aflatoxins-Biochemistry and Molecular Biology pp 397 - 414. Intech Publishing, Rijeka-Croatia.

Molina, A., Granados-Chinchilla, F., Jiménez, M., Acuña-Calvo, M.T., Alfaro, M, Chavarría,G. 2015. Salmonella vigilance of feedstuffs available in Costa Rica: Prevalence, serotyping and tetracycline resistance of isolates obtained from 2009 to 2014.Foodborne Pathogens and Disease. En prensa 
Molina y Granados. Inocuidad microbiológica de los alimentos para animales en Costa Rica

Norwegian Scientific Committee for Food Safety. 2006. Assessment of the risk from Salmonella occurring in feedingstuffs and the feed production process.

Pennington, J.H., Brooksbank, N. H., Pool, P.M., Seymour, F. 1968. Salmonella Virchow in a chicken-packing station and associated rearing units. BMJ 4:804-806.

Rivera-Pérez, W., Barquero-Calvo, E., Zamora-Sanabria, R. 2014. Salmonella Contamination Risk Points in Broiler Carcasses during Slaughter Line Processing. J. Food Protect. 77:2031-2034.

Sanli, T., Deveci, O., Sezgin, E. 2012. Effects of Pasteurization and Storageon Stability of Aflatoxin $M_{1}$ in Yogurt. The Journal of the Faculty ofVeterinary Medicine, University of Kafkas 18:987-990.

Scallan, E., Hoekstra, R.M., Angulo, F.J., Tauxe, R.V., Widdowson, M-A., Roy, S.L., 2011. Foodborne illness acquired in the United States-major pathogens. Emerg Infect Dis. 17:7-15.

Stolker, A.A.M, Manti, V., Zuidema, T., van Egmond, H., Deckers, E.R., Herbes, R., Hooglugt, J., Heuvel, E.O., de Jong, J. (2013). Carry-overof veterinary drugs from medicated to non medicated feeds in commercial feed manufacturing plants. Food Addit Contam: Part A. 30:1100-1107.

Sultana, N.,Hanif, N.Q. 2009. Mycotoxin contamination in cattle feed and feed ingredients. Pak. Vet. J., 29:211-213.

Tucker, W.B., Hogue, J.F., Adams G.D., Asiam, M., Shin L.S., Morgan G. 1992. Influence of dietary cation-anion balance during the dry period on the occurrence of parturient paresis in cows fed excess calcium. J. Anim. Sci. 70: 1238-1250.

Veldman, A., Vahl, H.A., Borggreve, G.J., Fuller, D.C. 1995. A survey of the incidence of Salmonella species and Enterobacteriaceae in poultry feeds and feed components. Vet. Rec. 136:169-172.

World Health Organization (WHO). 1997. Food safety and foodborne diseases. World Health Statistics Quarterly, Volume 50.

World Health Organization (WHO). 2013. Drug-resistant Salmonella. Fact sheet №139. Disponible en: http://www.who.int/mediacentre/factsheets/fs139/en/ 